\title{
AN IMMERSED BOUNDARY METHOD BASED FLUID-STRUCTURE INTERACTION SOLVER WITH APPLICATIONS IN ENERGY HARVESTING
}

\author{
GAURAV SHARMA ${ }^{1}$ AND RAJNEESH BHARDWAJ ${ }^{2}$ \\ ${ }^{1}$ Department of Mechanical Engineering, Indian Institute of Technology Bombay \\ Mumbai 400076 India \\ 184104006@iitb.ac.in \\ ${ }^{2}$ Department of Mechanical Engineering, Indian Institute of Technology Bombay \\ Mumbai 400076 India \\ rajneesh.bhardwaj@iitb.ac.in
}

Key words: Sharp-interface immersed boundary method, Large-scale flow-induced deformation, Vortexinduced vibration, Galloping, Energy harvesting.

\begin{abstract}
We present the development of an in-house fluid-structure interaction (FSI) solver and employ the solver for state-of-the-art applications in energy harvesting. An implicit partitioned approach is utilized to couple a sharp-interface immersed boundary method based flow solver and a finite-element method based structural solver. The code validations are presented for large-scale flow-induced deformation and vortex-induced vibration of an elastically mounted circular cylinder. We employ the FSI solver for analysis of vortex-induced vibration (VIV) of a cylinders, with different cross-sections. The suppression and agitation of VIV for different cylinders are discussed along with lock-in characteristics. An energy harvesting model is utilized to estimate the power generated per unit mass and it was found that the galloping of the D-cylinder is useful for broadband energy harvesting for a wide range of reduced velocities.
\end{abstract}

\section{INTRODUCTION}

Flow-induced vibration (referred as FIV hereafter) is an area of interest for many researchers due to its ubiquitous presence in several engineering problems. Most of the applications include FIV suppression to prevent the possible catastrophic damages being caused on the system. In the last decade, another application of FIV in wind and water based energy harvesting has been studied. The conventional wind energy harvesters or wind turbines operate in a very confined range of wind speeds (3 to $25 \mathrm{~m} / \mathrm{s}[1]$ ). Despite being highly efficient, the logistics constraints and the hazardous effects on the residing living beings[2], limit the use of wind turbines in very narrow region of the wind velocity. Similarly, the most commonly used water based energy harvesters i.e., hydroelectric power plants, mostly depend on the reservoir creation over a flowing water body, which alters surrounding ecosystem. An FIV based system does not require stagnating flowing water body and has relatively less moving parts, it may overcome the previously mentioned limitations. Another important application of FIV based devices is improve the convective heat transfer. The vortices produced during FIV could interact with heated wall and improve 
the transfer by reducing the thermal boundary layer thickness [3].

In the context of the energy-harvesting, Rostami and Armandei [4] have extensively reviewed the energy harvesting potential of FIV based systems. The energy is harnessed from the FIV system, primarily, in two ways. First way is using an electromagnetic coil mounted on the vibrating body to harvest electrical energy from the relative motion of the magnet and coil [5]. A second way is mounting piezoelectric energy harvester on the surface of the deforming body [6]. A comparative study done by Soti et al. [7] shows a very good agreement of average power for the constant damping energy harvesting model, with a more complex electromagnetic energy harvesting model. Therefore, for a simplistic analysis, energy harvesting potential of the systems has been investigated using the constant damping energy harvesting model. Bearman, in his notable review [8], concluded that a study of various different shapes is necessary to explore the complete potential of FIV system. Additionally, the study by Zhang et al. [9] shows significant improvement in energy harvesting potential with changing shapes of the elastically mounted structure. Therefore, the present study focuses on the the energy harvesting potential of various cross-sections. The comparison of energy conversion efficiency and power extraction has been performed to illustrate the best cross-section of the body, based on the specific applications and constraints. The simulations are performed at $R e=200$, assuming a two-dimensional flow.

In order to computationally tackle the above-mentioned problems, immersed boundary method is a good candidate and challenging problems including those with large-scale flow-induced deformation. Such cases have been successfully simulated with reasonable fidelity in previous studies [10]. In the present work, we employ a validated fluid-structure interaction (FSI) solver to demonstrate the energy harvesting

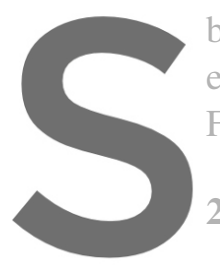
by elastically mounted dircular, D-section and inverted D cylinder. Firs
equations and code validations of the FSI solver (section 2 and 3) and
FIV response and associated energy harvesting potential (section 4)
2 GOVERNIN EQUATIONS

The present study utilizes an in-house sharp-interface immersed boundary method based Fluid-Structure

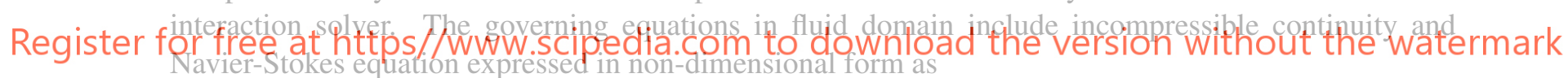

$$
\begin{gathered}
\frac{\partial u_{i}}{\partial x_{i}}=0 \\
\frac{\partial u_{i}}{\partial t}+u_{j} \cdot \frac{\partial u_{i}}{\partial x_{j}}=-\frac{\partial p}{\partial x_{i}}+\frac{1}{\operatorname{Re}} \frac{\partial^{2} u_{i}}{\partial x_{j} \partial x_{j}}
\end{gathered}
$$

The structual vibration is modeled using single degree of freedom approximation in non-dimensional form as

$$
\ddot{y}+\frac{2 \pi \xi}{U r} \dot{y}+\frac{4 \pi^{2}}{U r^{2}} y=\frac{F^{*}}{m^{*} A_{s}^{*}}
$$

The central difference discretized equations are solved using a fractional step Van-Kan method. The convective term is solved using Crank-Nicolson scheme and the diffusive term is solved using AdamBahsforth method. The pressure poisson equation is solved using the geometric multigrid method. A oneway coupling is introduced from fluid to structural using a sharp-interface immersed boundary coupling. 


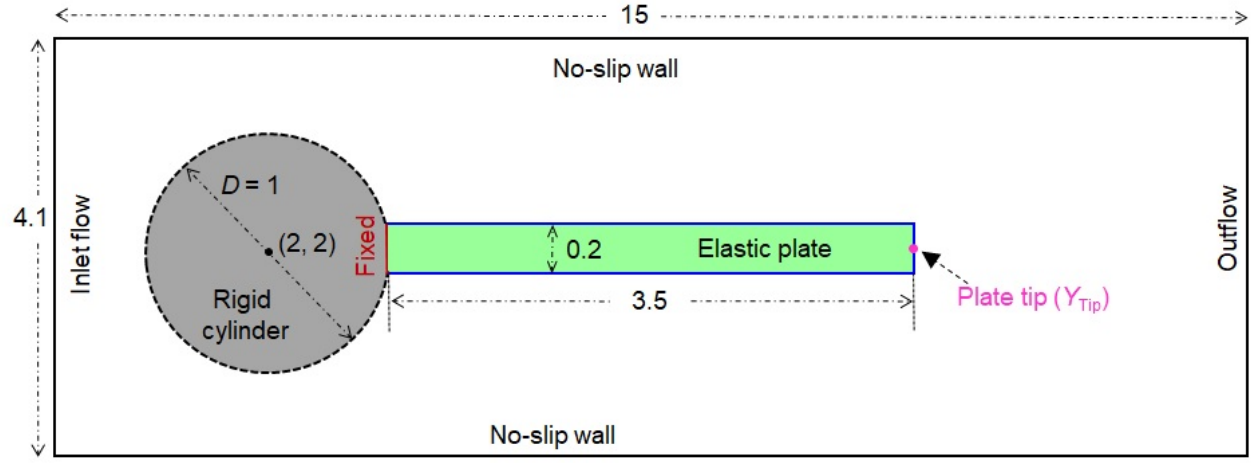

Figure 1: Schematic of the proposed FSI benchmark by Turek and Hron [17].

The structural equations are solved using Eulers method, and flow field is undated with new structural boundary coordinates. The details of computational method can be found in [11, 12]. The method has been extended for large scale deformations [13] and thermal buoyancy effects [14] during fluid structure interaction. An implicit partitioned approach is utilized to couple a sharp-interface immersed boundary method based flow solver and a finite-element method based structural solver. The implicit coupling has been accelerated using a dynamic under-relaxation scheme [15].

\section{CODE VERIFICATION}

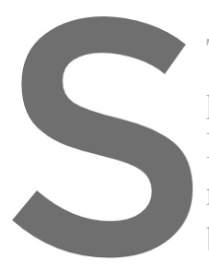

The in-house solver has been verified for flexible
previous studies $[15,16]$, the solver was yalidated
Hron [17], in which a thin, finite thickness, elastic
rigid cylinder, subjected to laminar flow (Figure 1).

bers $(R e)$, based on cylinder dianeter is 100 and the
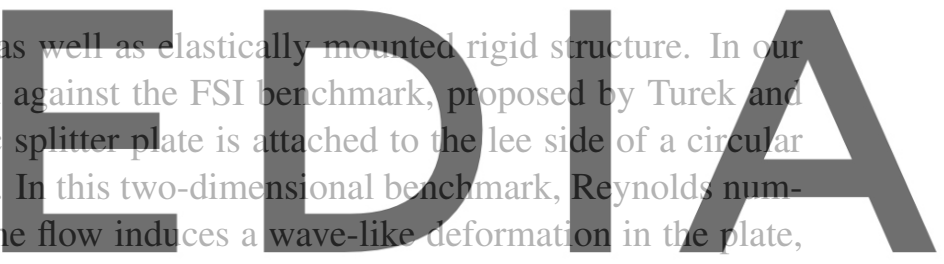

and it attains a periodic self-sustained oscillation. The comparison of computed time-varying displace-

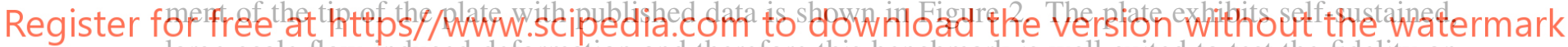

large-scale flow-induced deformation and therefore this benchmark is well-suited to test the fidelity on

an FSI solver. Next, an elastically mounted circular cylinder placed in a cross-flow, in the presence of a constant damping. The maximum displacement amplitude, lift force and phase difference are plotted in Figure 3 and show good agreement with the results of Leontini et al. [18].

\section{RESULTS AND DISCUSSION}

Elastically mounted cylinders of different cross-sections, namely, circular, D-section and inverted Dsection have been considered at $R e=200$ with constant mass ratio $m^{*}=10$. Reduced velocity $U r$ has been varied in the range of $[2,11.5]$. The domain size of $47 D \times 30 D$ and grid size of $0.02 D$ have been selected for the current study. This size is based on the previous studies [19,3], performed on similar systems and utilizing the same in-house solver. The structure is located at $15 D \times 15 D$ from the bottom left corner of the considered domain (Figure 4). The left wall is imposed with uniform inlet flow with velocity $u=1$. The top and bottom wall are imposed with free slip boundary conditions to approximate an open domain flow. The right wall is imposed with a developed flow or convective boundary condition. The simulation setup along with boundary conditions is shown in Figure 4. 


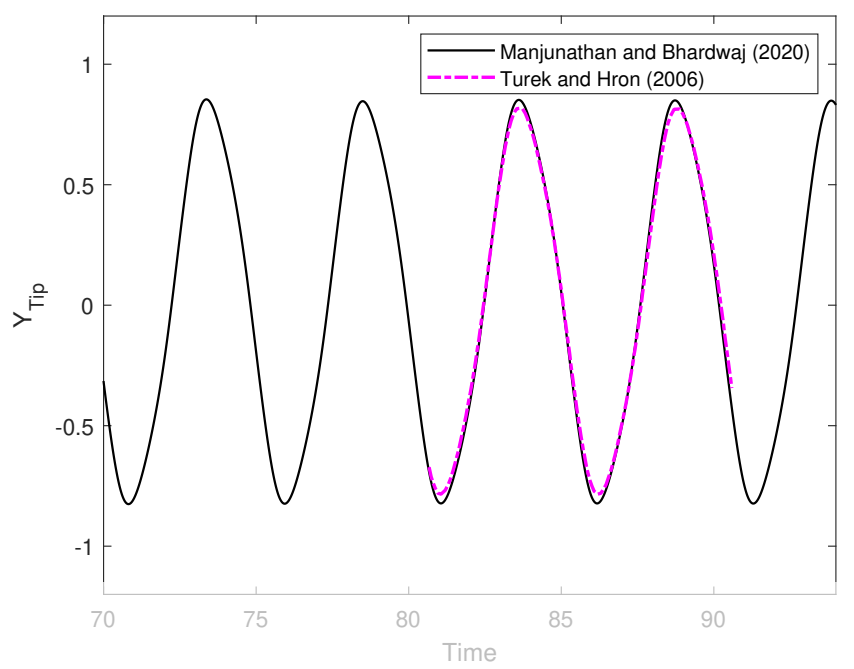

Figure 2: Comparison of computed displacement of the tip of the elastic plate with data of Turek and Hron [17]. Adapted from Manjunathan and Bhardwaj [16].
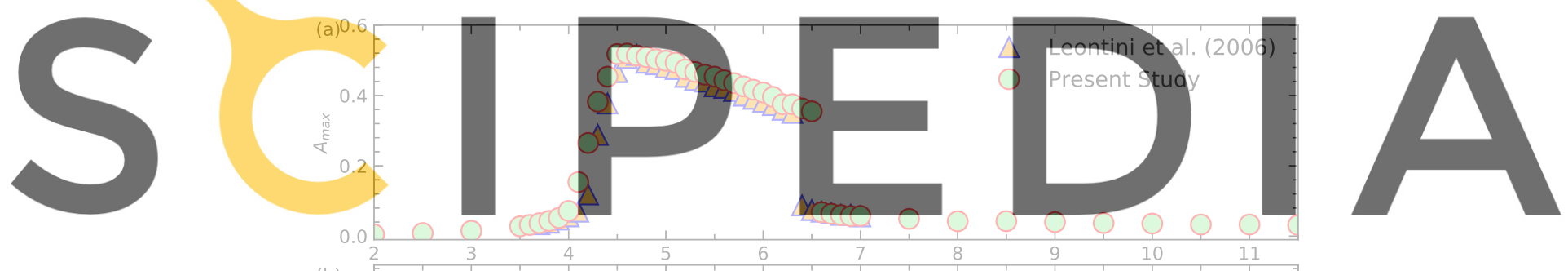

Register for free at hateps//www.scipedia.com to download the version without the watermark

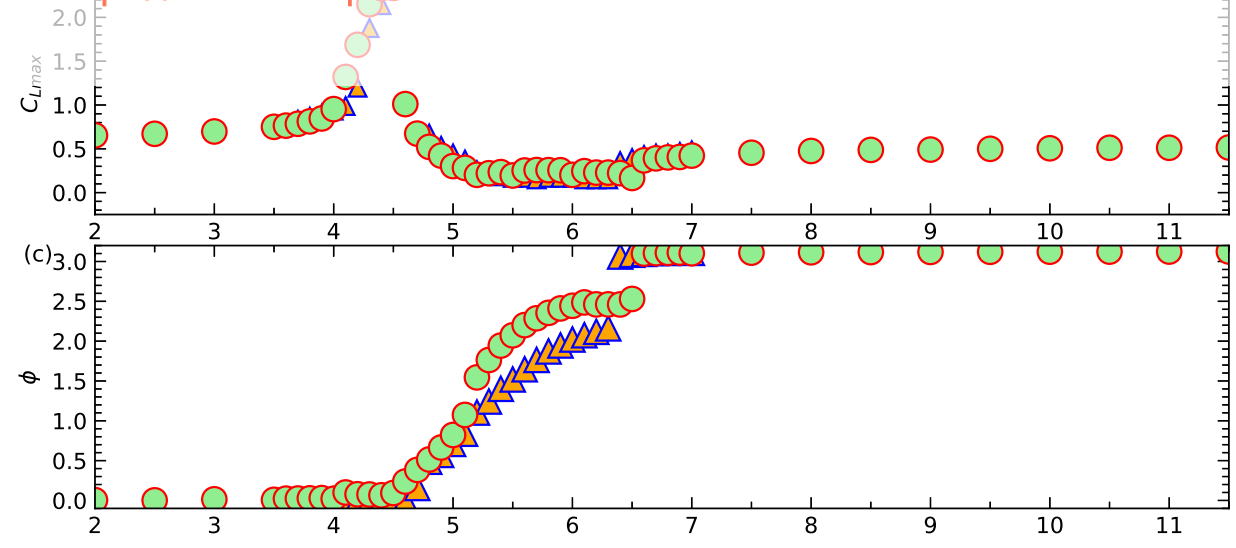

Figure 3: Comparison of (a) $A_{\max }$, (b) $C_{L \max }$ and (c) $\phi$ calculated using in-house solver for circular cylinder at $R e=200, m^{*}=10$ and $\xi=0.01$ with data of Leontini et al. [18]. 


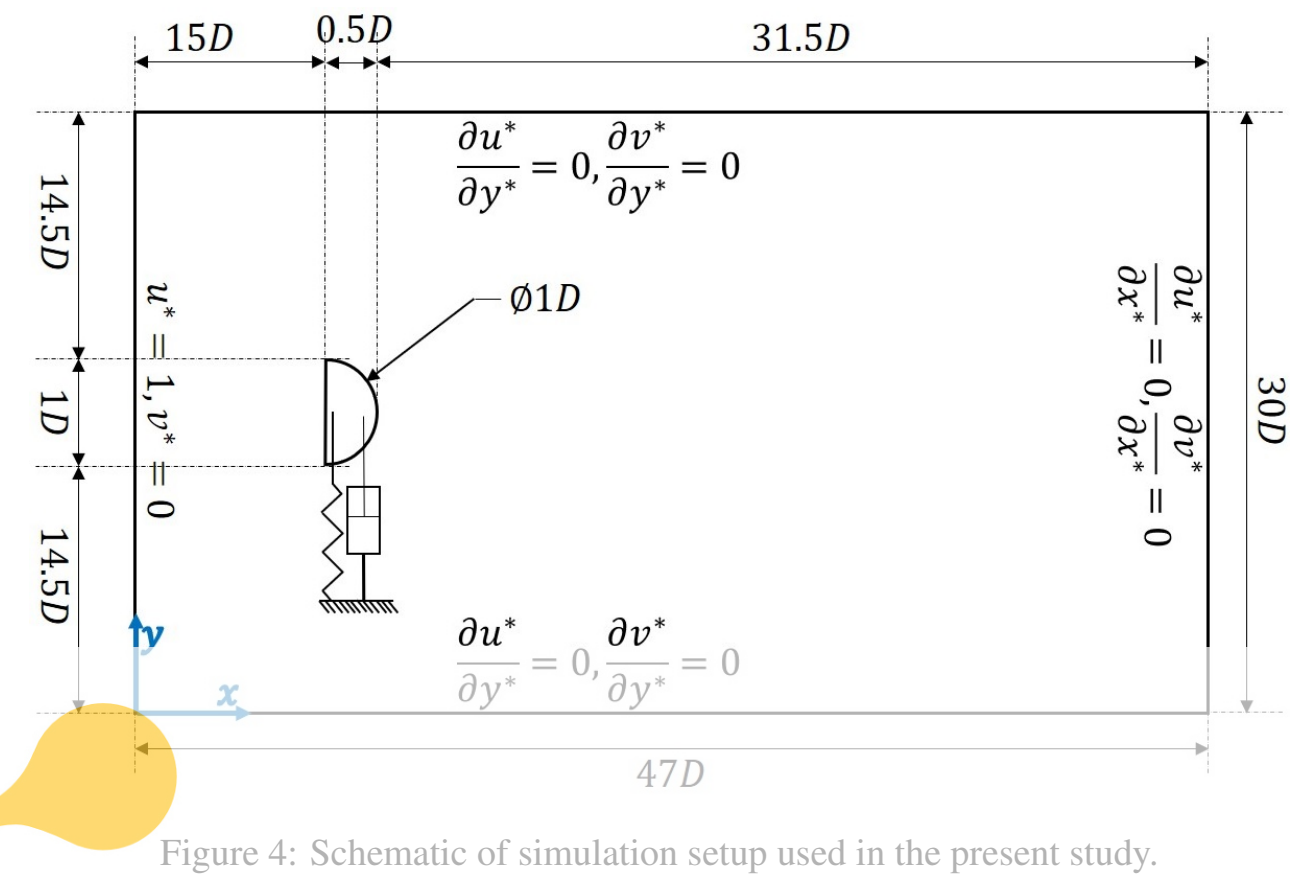

The maximum amplitude of the vibration of various cross-sections has been plotted with varying reduced

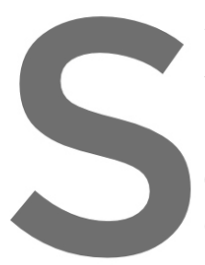
velocities in Figure 5. The D section cylindex to inverted D and circular [20]. Additionally, the i. cylinder. This implies of structural material.
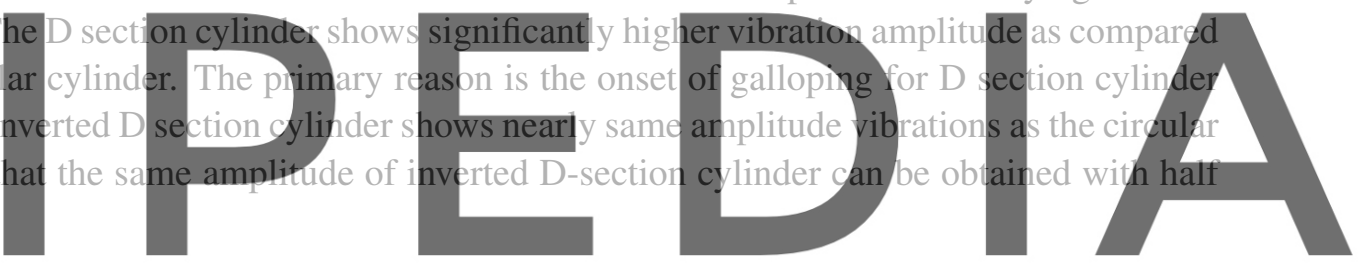

The non-dimensional power output shows maximum power generation for circular cylinder, despite its

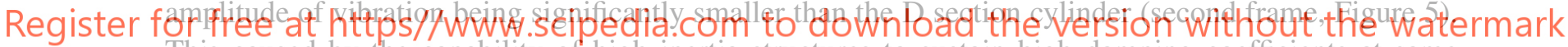
This caused by the capability of high inertia structures to sustain high damping coefficients at same

damping ratio. However, a higher power generation per unit mass is observed for D-section cylinder of

the structural material (third frame, Figure 5). This aspect is important if material cost is significant. Additionally, the reduction in power generated by D-section cylinder is much lower with increasing $U r$, as compared to the circular cylinder. This implies that the D-section cylinder could be used as a broadband energy-harvester. The inverted D section cylinder generates similar power per unit mass as the circular cylinder. Further, the energy conversion efficiency is computed per unit fluid power present in the area swept by the structure (fourth frame, Figure 5). While D-section cylinder exhibits the largest amplitude of vibration, the efficiency is not the largest and lower than that for the circular cylinder. The energy efficiency is the highest for the circular cylinder for $4.5<U r<6.5$ and D-section provides a reasonably larger efficiency over a wide range of reduced velocity.

\section{CONCLUSIONS}

We have reported the development of an fluid-interaction interaction (FSI) solver based on sharp-interface immersed boundary method and finite-element method. The code validations were carried for large-scale 

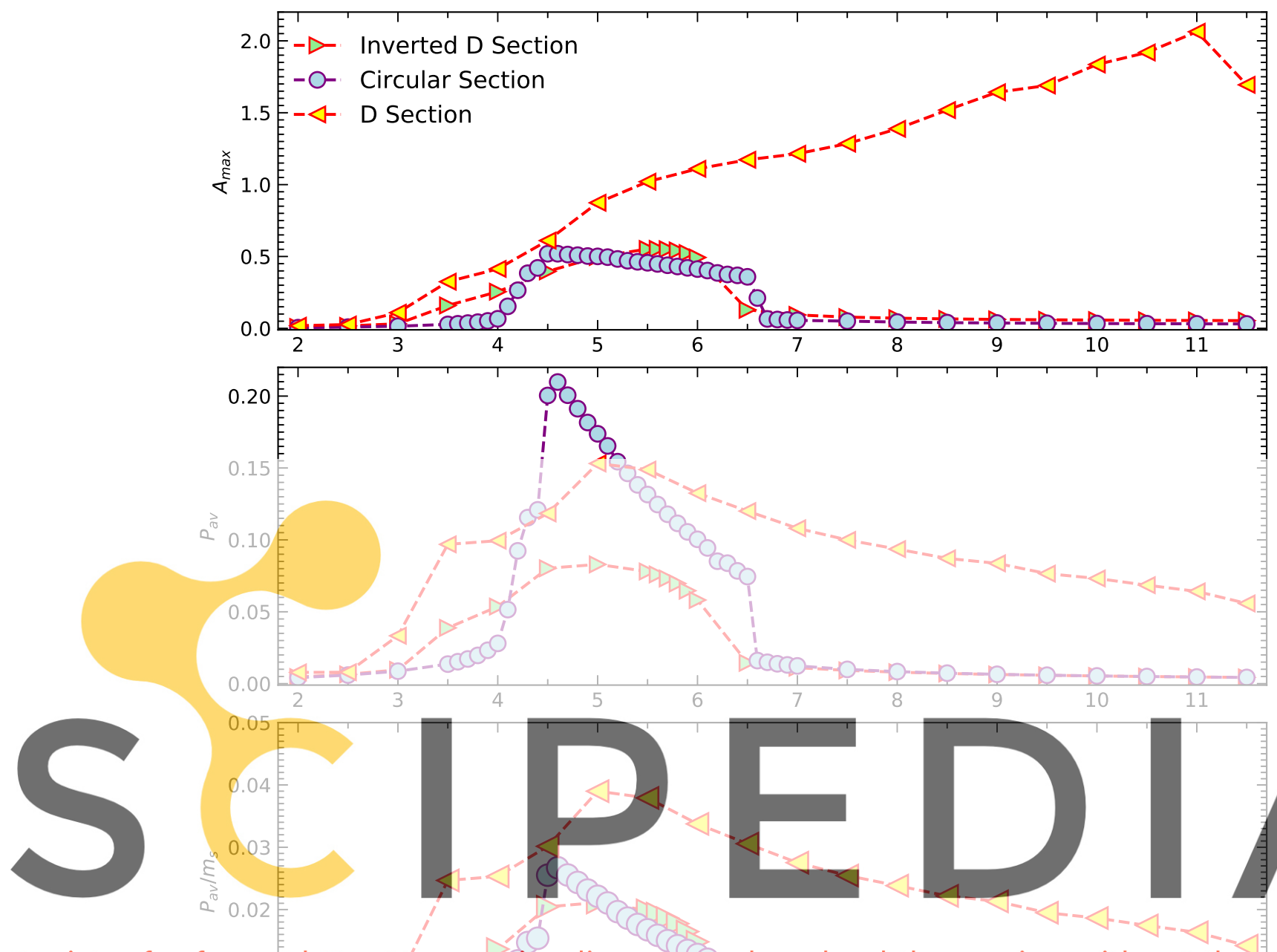

Register for frees at https/Lwww.scipedia.com to download the version without the satermark
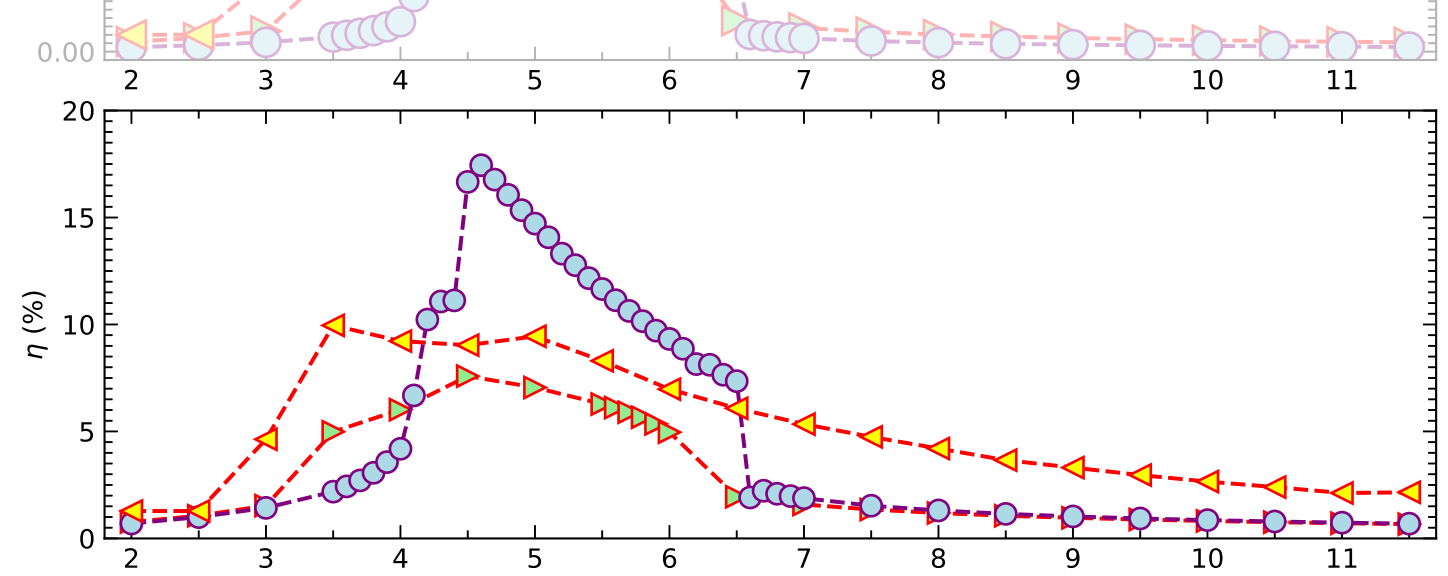

Figure 5: Amplitude response (first frame), power output (second frame), power output per unit structure mass (third frame) and energy conversion efficiency (fourth frame) of the different cross-section cylinders considered in the present work. 
flow-induced deformation of a splitter plate and vortex-induced vibration of a elastically mounted cylinder on a linear spring in laminar flow. These tests demonstrate the fidelity of the solver proposed. The solver has been employed to understand the flow-induced vibration response of elastically mounted cylinders different cross-sections. We have found that the D-section cylinder shows significant increase in the amplitude for a wide range of reduced velocity as compared to circular and inverted D-cylinder. The large amplitude vibration at larger reduced velocity for the D-section cylinder is attributed to galloping behaviour. The cylinders were tested for the potential of energy harvesting and D-section was found to generate the largest power per unit mass for a wide range of the reduced velocity, among the three cylinders considered. Our study provides insights while designing broadband energy harvester capable for high power generation.

\section{ACKNOWLEDGEMENTS}

We gratefully acknowledge financial support by a grant (Grant No. MTR/2019/000696) from the Science and Engineering Research Board (SERB), Department of Science and Technology (DST), New Delhi, India.

\section{REFERENCES}

[1] Abed, KA and El-Mallah, AA, Capacity factor of wind turbines, Energy (1997) 22(5):487-491.

[2] Broner, Nick, The effects of low frequency noise on people - a review, Journal of Sound and Vibration (1978) 58(4):483-500.
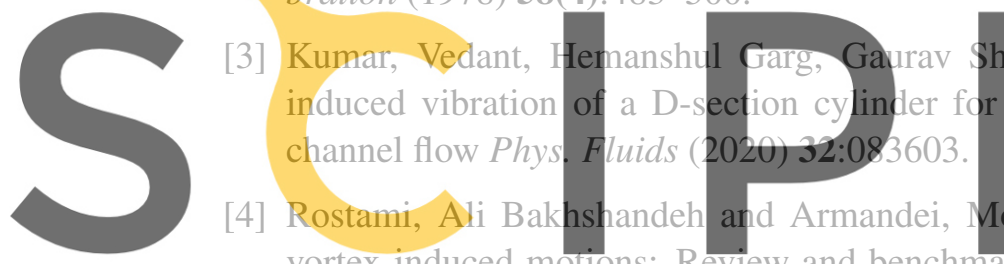
vortex-induced motions: Review and benchmarking of technologies, Renewable and Sustainable Energy Reviews (2017) 70:193-214.

Register for free at https//www.scipedia.com to download the version without the watermark

[5] Dai, HL and Abdelkefi, A and Javed, U and Wang, L, Modeling and performance of electromagnetic energy harvesting from galloping oscillations, Smart Materials and Structures (2015) 24(4):045012.

[6] Zhang, LB and Abdelkefi, A and Dai, HL and Naseer, R and Wang, L, Design and experimental analysis of broadband energy harvesting from vortex-induced vibrations Journal of Sound and Vibration (2017) 408:210-219.

[7] Soti, Atul Kumar and Thompson, Mark C and Sheridan, John and Bhardwaj, Rajneesh, Harnessing electrical power from vortex-induced vibration of a circular cylinder Journal of Fluids and Structures (2017) 70:360-373.

[8] Bearman, Peter W, Vortex shedding from oscillating bluff bodies AnRFM (1984) 16:195-222.

[9] Zhang, Baoshou and Song, Baowei and Mao, Zhaoyong and Tian, Wenlong and Li, Boyang, Numerical investigation on VIV energy harvesting of bluff bodies with different cross sections in tandem arrangement Energy (2017) 133:723-736.

[10] Bhardwaj, Rajneesh, Vortex shedding from oscillating bluff bodies In Immersed Boundary Method 
(Eds.: Roy et al.) (2020) Springer, Singapore.

[11] Mittal, Rajat and Dong, Haibo and Bozkurttas, Meliha and Najjar, FM and Vargas, Abel and Von Loebbecke, Alfred, A versatile sharp interface immersed boundary method for incompressible flows with complex boundaries Journal of computational physics (2008) 227(10):4825-4852.

[12] Seo, Jung Hee and Mittal, Rajat, A sharp-interface immersed boundary method with improved mass conservation and reduced spurious pressure oscillations Journal of computational physics (2011) 230(19):7347-7363.

[13] Bhardwaj, Rajneesh and Mittal, Rajat, Benchmarking a coupled immersed-boundary-finite-element solver for large-scale flow-induced deformation AIAA journal (2012) 50(7):1638-1642.

[14] Garg, Hemanshul and Soti, Atul K and Bhardwaj, Rajneesh, A sharp interface immersed boundary method for vortex-induced vibration in the presence of thermal buoyancy Physics of Fluids (2018) 30(2):023603.

[15] Kundu, Anup, Atul K. Soti, Hemanshul Garg, Rajneesh Bhardwaj, and Mark C. Thompson, Computational modeling and analysis of flow-induced vibration of an elastic splitter plate using a sharpinterface immersed boundary method SN Applied Sciences (2020) 2:1110.

[16] Manjunathan, Sujyesh Aanandh, and Rajneesh Bhardwaj, Thrust generation by pitching and heaving of an elastic plate at low Reynolds number Phys. Fluids (2020) 32:073601.

[17] Turek, Stefan and Hron, Jaroslav, Proposal for numerical benchmarking of fluid-structure interac-

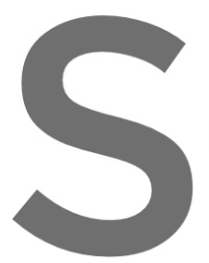
tion between an elastic (2006):371-385.

[18] Leontini, Justin Scott and branching behavidur and Structures (2006) 22(6-7):857-864.
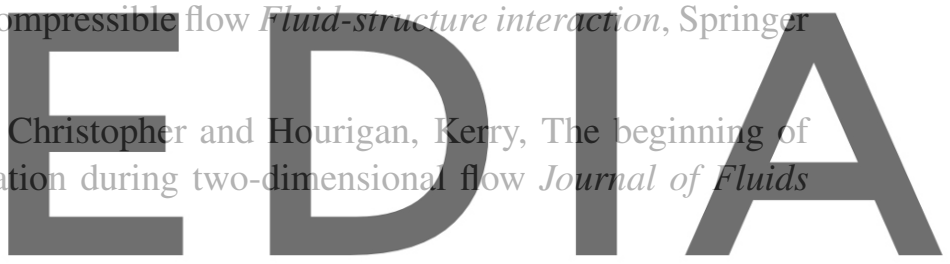

[19] Garg, Hemanshul and Soti, Atul Kumar and Bhardwaj, Rajneesh, Vortex-induced vibration and

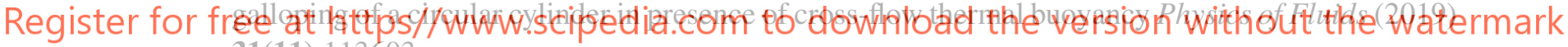
31(11): 113603 .

[20] Zhao, J and Hourigan, $K$ and Thompson, MC, Flow-induced vibration of D-section cylinders: an afterbody is not essential for vortex-induced vibration Journal of Fluid Mechanics (2018) 851:317343. 